\title{
Quantum simulation of Anderson and Kondo lattices with superconducting qubits
}

\author{
Juan José García-Ripoll, ${ }^{1}$ Enrique Solano, ${ }^{2,3}$ and Miguel Angel Martin-Delgado ${ }^{1}$ \\ ${ }^{1}$ Universidad Complutense, Facultad de Físicas, Ciudad Universitaria s/n, Madrid, E-20808, Spain \\ ${ }^{2}$ Physics Department, CeNS and ASC, Ludwig-Maximilians-Universität, Theresienstrasse 37, 80333 Munich, Germany \\ ${ }^{3}$ Sección Física, Departamento de Ciencias, Pontificia Universidad Católica del Perú, Apartado 1761, Lima, Peru
}

\begin{abstract}
We introduce a mapping between a variety of superconducting circuits and a family of Hamiltonians describing localized magnetic impurities interacting with conduction bands. This includes the Anderson model, the single impurity one- and two-channel Kondo problem, as well as the 1D Kondo lattice. We compare the requirements for performing quantum simulations using the proposed circuits to those of universal quantum computation with superconducting qubits, singling out the specific challenges that will have to be addressed.
\end{abstract}

\section{INTRODUCTION}

Quantum simulation ${ }^{1}$ consists on tuning the dynamics of a flexible quantum mechanical system to simulate the properties of another physical system or of a quantum mechanical model whose solution is unknown. While a universal quantum computer can efficiently simulate the dynamics of any quantum system ${ }^{2 / 3}$, designing a quantum simulator for a specific model should simplify the experimental requirements. In particular, quantum simulators require neither high fidelity thresholds nor error correction, and thus fewer qubits are needed to get interesting results which are out of reach for classical numerical computations. The successful simulation of the BoseHubbard model using cold atoms in optical lattices 45 is a paradigmatic example.

In the last years, there have been tremendous experimental achievements in the context of superconducting qubits, improving both quantum control and coherence times $6 / 7 / 8|9| 10|11| 12|13| 14 \mid 15$. While the aim is the implementation of a scalable universal quantum computer $\frac{14115 \mid 16}{}$, this technology can be used in the quantum simulation of many-body physics with JosephsonJunction arrays $17 / 18 \mid 19$.

With this motivation, we first design a circuit to simulate the Anderson model for an impurity ion coupled to conduction electrons 20 . This is done through an exact mapping from the effective models describing the superconducting circuits to the fermionic Hamiltonian for the impurity problem. In a certain parameter regime of our circuit, this leads to the Kondo Hamiltonian modeling the non-trivial physics of itinerant electrons that interact with a localized impurity having non-zero magnetic moment ${ }^{21}$. This is one of the central models for strongly correlated electrons with implications going beyond Condensed Matter Physics. Furthermore, our constructions are versatile enough to simulate other relevant Hamiltonians. With an appropriate choice of the geometry, these include the Kondo lattice ${ }^{22}$ and the multichannel Kondo mode 23 . The latter exhibits the simplest example of non-Fermi liquid behavior, which is a strong deviation from the standard model of condensed matter systems introduced by Landau.

We will also discuss the experimental challenges for im- plementing such circuits. In particular, while coherence and interaction times of current superconducting charge qubits seem good enough to simulate the long-time dynamical and static properties of these lattices, further experimental work is needed. Finally, we propose a set of measurements to gather information about transport, correlation and energy spectra and comment possible implications of the present work.

\section{CIRCUIT DESIGN}

\section{A. Elementary components}

Let us start by describing the proposed architecture. We consider two or more lines of low-capacitance superconducting islands coupled by Josephson junctions [Fig. 17]. While not shown here, each island is itself part of a circuit like that of a charge qubit, with an external lead which capacitively induces an offset potential on the island, and possibly a Josephson coupling to a superconducting reservoir of Cooper pairs 24 .

Our quantum simulation protocols are based on two building blocks. First, as we will show below, each island will have a very low capacitance and be constrained to have at most one Cooper pair, which can be treated as an impenetrable boson. Second, selected pairs of islands will be coupled capacitively [Fig. 1p]. The coupling and voltages of these islands will be adjusted to create a suitable energy landscape that favors having a single excess Cooper pair in either the upper or lower island, states which we associate with an effective pseudo spin. The appropriate choice of energies has the following form

$$
E\left(n_{0 \uparrow}, n_{0 \downarrow}\right)=U n_{0 \uparrow} n_{0 \downarrow}-\epsilon\left(n_{0 \uparrow}+n_{0 \downarrow}\right) .
$$

Here, $n_{0 \uparrow}$ and $n_{0 \downarrow}$ represent the excess of Cooper pairs on the upper and lower island in the impurity [Fig. 1 1 -b] The constants $U$ and $\epsilon$ denote, respectively, the repulsive interaction energy coming from the capacitive coupling between islands and an energy offset of these islands with respect to the rest of the circuit.

As mentioned before, when we embed such an element in a circuit, the energy offset $\epsilon$ enforces the impurity to host at most a single excess Cooper pair. In Fig. 11 we 


\section{a)}

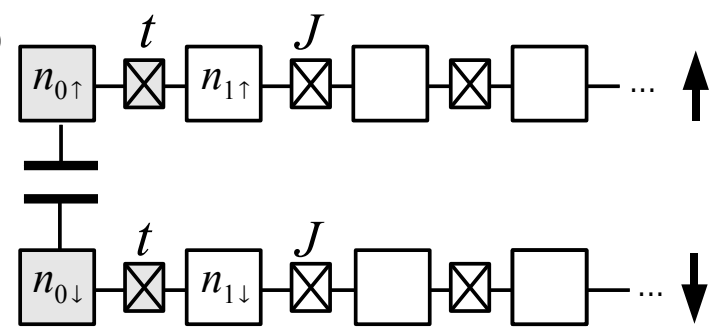

b)

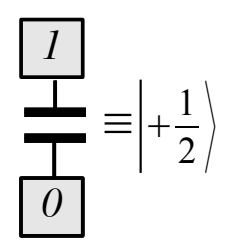

c)
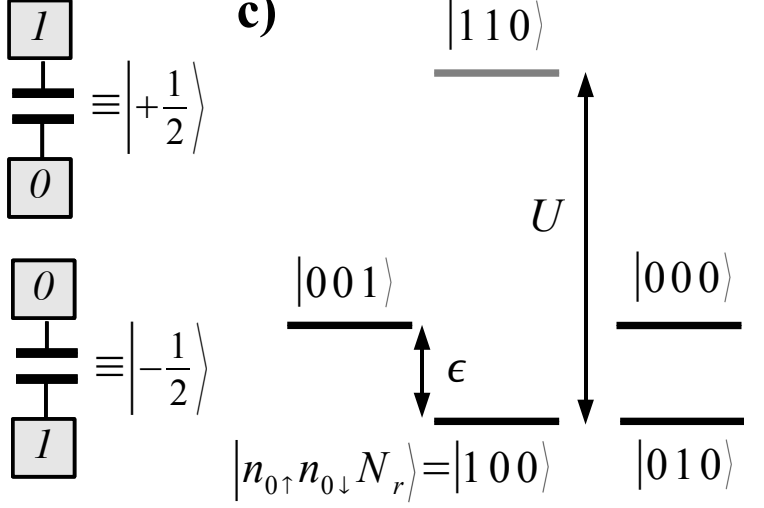

FIG. 1: (a) Quantum circuit simulating the single Anderson or Kondo impurity model. The big square boxes are superconducting islands, connected by crossed squares which are Josephson junctions. A selected pair of islands is capacitively coupled and represents a magnetic impurity. The Josephson energy for tunneling between junctions are $J$ for the main lattice and $t$ around the impurity. (b) Two occupation states for the impurity representing a pseudo-spin state. (c) Energy levels of the impurity and a neighboring site. $n_{0 \uparrow}, n_{0 \downarrow}$ and $N_{r}$ represent the number of pairs of the two islands in the impurity and of the rest of the circuit. By external voltages, $-\epsilon / 2$, and the capacitive coupling, $U \sim 1 / C$, it becomes favorable to have a single pair in the impurity.

have depicted the lowest energy levels of a circuit containing a single impurity, with occupation numbers $n_{0 \uparrow}$ and $n_{0 \downarrow}$, connected to an array of ordinary islands, with total population $N_{r}$. A possible configuration is shown in Fig. 17. If we neglect the kinetic energy of hopping pairs, we find that the states where the impurity is occupied by a single pair, $n_{0 \uparrow}=1$ or $n_{0 \downarrow}=1$, have lower energy than states where this pair is in the rest of the circuit. Multiply occupied impurities, $n_{0 \uparrow}+n_{0 \downarrow}>1$, have a even larger energy, $U$, so that we can neglect these states.

\section{B. Quantum circuit model}

In Sect. IID through II G we will combine the previous elements in circuits that simulate specific Hamiltonians. In order to perform this task we must first introduce the mathematical description of such circuits and the approximations under which they will operate. We start considering the standard Hamiltonian $\left.{ }^{18} 19\right]$ describing the physics of a Josephson junction array such as the one in Fig. 1 a

$$
\begin{aligned}
H & =\sum_{i j, \sigma=\uparrow, \downarrow} \frac{1}{2}\left(n_{i \sigma}-n_{i \sigma}^{(g)}\right) \bar{U}_{i j}\left(n_{j \sigma}-n_{j \sigma}^{(g)}\right) \\
& +\sum_{i j, \sigma=\uparrow, \downarrow} \bar{J}_{i j} \cos \left(\phi_{i \sigma}-\phi_{j \sigma}\right)+E\left(n_{0 \uparrow}, n_{0 \downarrow}\right) .
\end{aligned}
$$

Here, $n_{i \sigma}$ and $\phi_{i \sigma}$ are conjugate variables denoting the excess number of Cooper pairs on an island and the associated superconducting phase, respectively. $J_{i j}$ is the Josephson energy for a junction that connects two islands. Finally, there is a short range interaction term, $\bar{U}=4 e^{2} C^{-1}$, which is the inverse of the capacitance matrix. Similar to $\bar{J}, C$ is a sparse matrix with nonzero elements on the diagonal, denoting the intrinsic capacitance of each island, $C_{i i} \neq 0$, and also between islands connected by junctions or capacitors. We choose all capacitances to be small, $4 e^{2} / C \gg \bar{J}$, and impose $C_{i i} \gg C_{i \neq j}$ so that we can neglect any off-site interaction, $\bar{U}_{i \neq j} \simeq 0$.

The residual charges, $n_{i}^{(g)}=n_{i}^{(0)}+n_{i}^{(D C)}$, are a combination of a possible systematic shift, $n_{i}^{(0)}$, and an effective term $n_{i}^{(D C)}=C_{i}^{(g)} V_{i}^{(g)} / 2 e$, proportional to the potential applied to each island, $V_{i}^{(g)}$, and the capacitance through which it is applied, $C_{i}^{(g)}$ [See Ref. 24] From this it follows that the residual charges can be set to zero by appropriate tuning the offset voltages acting on each junction. As in current single and two-qubit experiments $78|11| 12 \mid 14$, we will work in the so called sweet spot of energetic degeneracy, given by $n_{i}^{(g)}=1 / 2$. Due to the low capacitance, $\bar{U} \gg \bar{J}$, the state of each island is then constrained to having 0 or 1 Cooper pair, or any linear superposition of these states.

Contrary to the cavity-QED and superconducting qubit experiments 14 we would like the total number of Cooper pairs to be a well defined quantum number. This means we do not want a residual coupling between the states with 0 and 1 pairs, which would correspond to a term $J_{i i} \cos \left(\phi_{i}\right)$ in the previous model. This is effectively achieved by switching off the coupling between the superconducting islands and the Cooper pair reservoir during the experiment.

Finally, it is interesting to note that the total number of pairs in the ground state can be tuned with very small voltages that take islands away from degeneracy and which play the role of a local chemical potential

$$
\mu_{i \sigma}=\sum_{j} \bar{U}_{i j}\left(n_{i \sigma}^{(g)}-\frac{1}{2}\right) .
$$

\section{Hard-core boson model}

Summarizing the previous approximations, we have an array of superconducting islands coupled both capacitively and via Josephson tunneling. At the same time 
we have ensured that each superconducting island has 0 o 1 excess Cooper pair, all other states being energetically unfavorable. In other words, we have imposed the hard-core bosons conditions on the Cooper pairs: they become like impenetrable bosonic particles such that no two pairs with the same effective "spin" the same site.

In this limit we can describe any configuration of the circuit using the occupation numbers $\left\{n_{i \sigma}\right\}$. Due to the hard-core condition, the dynamics is well approximated by the projection of the circuit Hamiltonian (2) onto the relevant energy subspace, $n_{i \sigma} \in\{0,1\}$. To perform this projection we will need to express the phase terms in basis of occupation numbers

$$
\begin{aligned}
& \sum_{i j \sigma} \bar{J}_{i j} \cos \left(\phi_{i \sigma}-\phi_{j \sigma}\right)= \\
& \sum_{i j \sigma} \frac{1}{2} \bar{J}_{i j} \sum_{n_{i \sigma}, n_{j \sigma}}\left|n_{i \sigma}+1, n_{j \sigma}-1\right\rangle\left\langle n_{i \sigma}, n_{j \sigma}\right|+\text { H.c. }
\end{aligned}
$$

Note that this equation has a very simple interpretation: Cooper pairs are transferred one by one between any two islands connected by the hopping matrix $\bar{J}_{i j}$.

The projection is implicitly performed by means of a more concise mathematical representation that includes an exclusion principle for particles on the same site. We introduce hard-core bosonic operators, $a_{i \sigma}^{\dagger}$ and $a_{i \sigma}$, which respectively create and annihilate a Cooper pair on a given site. They act on the basis elements as follows

$$
\begin{aligned}
a_{i \sigma}\left|n_{i \sigma}=1\right\rangle=\left|n_{i \sigma}=0\right\rangle, & a_{i \sigma}|0\rangle=0, \\
a_{i \sigma}^{\dagger}|0\rangle & =|1\rangle, \quad a_{i \sigma}^{\dagger}|1\rangle=0,
\end{aligned}
$$

have the usual bosonic commutation relations

$$
\left[a_{i \sigma}, a_{j \sigma^{\prime}}^{\dagger}\right]=\delta_{i j} \delta_{\sigma \sigma^{\prime}}, \quad\left[a_{i \sigma}, a_{j \sigma^{\prime}}\right]=0,
$$

and enforce the hard-core condition simply because $a_{i \sigma}^{\dagger 2}=$ 0 . Using these operators we can express the number of particles $n_{i \sigma}=a_{i \sigma}^{\dagger} a_{i \sigma}$ and the projected phase operator,

$$
2 \cos \left(\phi_{i \sigma}-\phi_{j \sigma}\right) \simeq a_{i \sigma}^{\dagger} a_{j \sigma}+a_{j \sigma}^{\dagger} a_{i \sigma},
$$

which adopts the form of a hopping term.

\section{Single impurity Anderson model}

We will apply all the approximations and techniques introduced before to study a particular circuit, which consists of two impurity islands coupled to two 1D Josephson junction arrays [Fig. 1 17]. The low energy dynamics of this circuit is described by the bosonic Hamiltonian

$$
\begin{aligned}
H_{A} & =-J \sum_{i \geq 1}\left(a_{i+1 \sigma}^{\dagger} a_{i \sigma}+\text { H.c }\right)-t \sum_{\sigma}\left(a_{0 \sigma}^{\dagger} a_{1 \sigma}+\text { H.c. }\right) \\
& +E\left(n_{0 \uparrow}, n_{0 \downarrow}\right)+\sum_{i \sigma} \mu_{i \sigma} n_{i \sigma} .
\end{aligned}
$$

Out of all terms in the hopping matrix $\bar{J}_{i j}$, we have left only the Josephson energies between sites in the conduction array, $\bar{J}_{i i+1}=J$ for $i>0$, and the coupling between the impurity site and this band, $\bar{J}_{01}=\bar{J}_{10}=t$ [See Fig. 17]. Finally, as mentioned in Sect. II C, we have included an effective chemical potential which depends on deviations from the degeneracy point of the local potentials acting on each island.

We can map this Hamiltonian to a fermionic model using another standard tool, the Jordan-Wigner transformation $25 \mid 26$

$$
\begin{aligned}
a_{i \uparrow} & =c_{i \uparrow}(-1)^{\sum_{j<i} n_{j \uparrow}}, \\
a_{i \downarrow} & =c_{i \downarrow}(-1)^{\sum_{j<i} n_{j \downarrow}+N_{\uparrow}}, \\
n_{i \sigma} & =c_{i \sigma}^{\dagger} c_{i \sigma}=a_{i \sigma}^{\dagger} a_{i \sigma} .
\end{aligned}
$$

The new operators satisfy the usual fermionic anticommutation relations $\left\{c_{i \sigma}, c_{j \sigma^{\prime}}^{\dagger}\right\}=\delta_{i j} \delta_{\sigma \sigma^{\prime}}$ and $N_{\sigma}=$ $\sum_{i} n_{i \sigma}$. With this transformation, our effective Hamiltonian (9) converts identically into a fermionic model

$$
\begin{aligned}
H_{A} & =-J \sum_{i \geq 1}\left(c_{i+1 \sigma}^{\dagger} c_{i \sigma}+\text { H.c }\right)-t \sum_{\sigma}\left(c_{0 \sigma}^{\dagger} c_{1 \sigma}+\text { H.c. }\right) \\
& +E\left(n_{0 \uparrow}, n_{0 \downarrow}\right)+\sum_{i \sigma} \mu_{i \sigma} n_{i \sigma}
\end{aligned}
$$

which is known as the Anderson impurity model20! We have thus demonstrated that the Anderson Hamiltonian accurately describes the dynamics of a circuit that we have designed, under reasonable approximations. Following the ideas sketched in the introduction, we now argue that the quantum circuit can itself be used to simulate the dynamical and static properties of the Anderson model. For instance, if we are able to create and cool this circuit to its ground state, we will create a state which is related to the ground state of the Anderson model by a unitary transformation. The properties of the equivalent fermionic system can be recovered from measurements of the Cooper pairs and the Jordan-Wigner relations (8).

In the following subsections we will show how, by choosing other limiting cases and designing slightly different circuits, one can simulate not only the Anderson model, but other more complicated and interesting Hamiltonians.

\section{E. Kondo model}

A special limit of the Anderson model is obtained when we impose a low capacitance on the impurity, $U \rightarrow \infty$, and make the energy displacement large compared to the coupling between the impurity and its neighbors, $t \ll$ $\epsilon$. Using standard second order perturbation theory, the result is the single impurity Kondo Hamiltonian ${ }^{32}$

$$
H_{K}=-J \sum_{\langle i, j\rangle \sigma} c_{i \sigma}^{\dagger} c_{j \sigma}+J_{K} \vec{S}_{0} \cdot \vec{s}_{1} .
$$



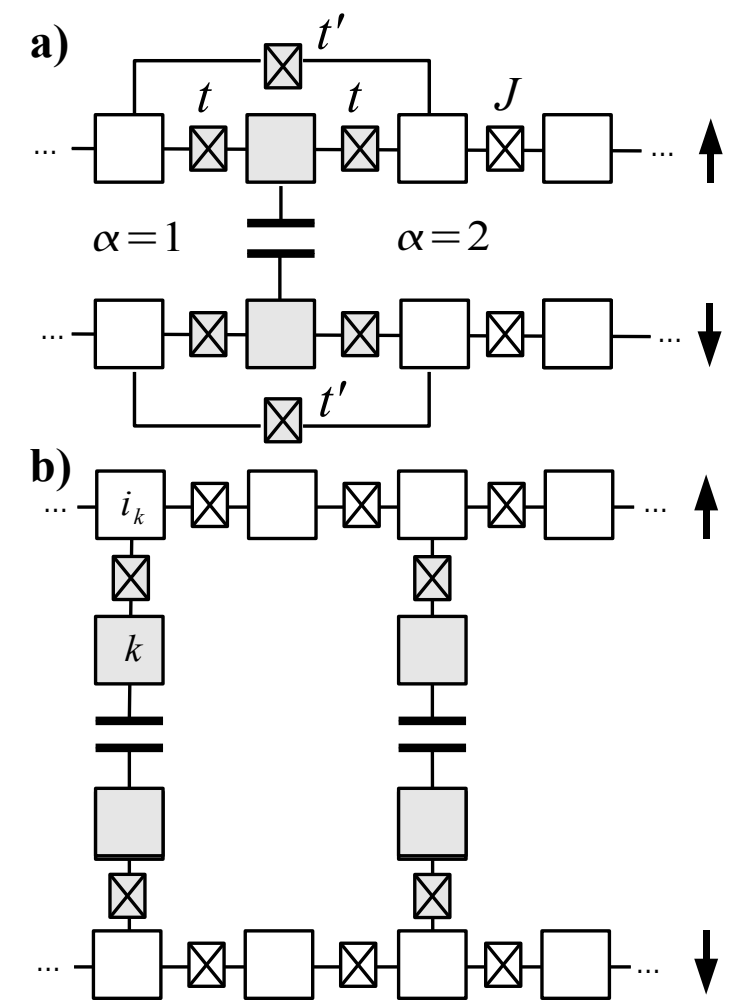

FIG. 2: (a) Circuit for a two-channel single impurity Kondo system. Each half of the circuit is associated to a different channel. An extra junction with negative sign, $t^{\prime} \simeq-J_{K}$, is a quantum interference element that prevents hopping between channels. (b) Circuit for a 1D Kondo lattice.

Here $\vec{S}_{0}$ and $\vec{s}_{1}$ are the pseudo-spins of the impurity and of the first lattice site

$$
\vec{S}_{0}=\sum_{\sigma, \sigma^{\prime}} \frac{1}{2} \vec{\sigma}_{\alpha \sigma^{\prime}} c_{0 \sigma}^{\dagger} c_{0 \sigma^{\prime}}, \text { and } \vec{s}_{1}=\sum_{\sigma, \sigma^{\prime}} \frac{1}{2} \vec{\sigma}_{\alpha \sigma^{\prime}} c_{1 \sigma}^{\dagger} c_{1 \sigma^{\prime}},
$$

respectively, and $J_{K}=t^{2} / \epsilon$ is the anti-ferromagnetic coupling between the Kondo impurity and the free fermions. We want to stress that by tuning the parameters $J, t$ and $\epsilon$, we can realize a crossover from a low energy regime below the Kondo temperature $T_{K}$ to a high energy limit.

\section{F. Two-channel Kondo model}

The previous setup and treatments can be reused to simulate a two-channel Kondo system 23 . As shown in Fig. $2 \mathrm{a}$, the impurity is in the middle of a one-dimensional lattice, coupled to two different conduction bands. Following similar steps, the new effective model becomes

$$
H_{K}=-J \sum_{\langle i, j\rangle \sigma, \alpha} c_{i \sigma \alpha}^{\dagger} c_{j \sigma \alpha}+J_{K} \sum_{\alpha=1,2} \vec{S}_{0} \cdot \vec{s}_{1 \alpha}
$$

where $\alpha \in\{1,2\}$ denotes the two fermionic channels of our problem. Note that in this Hamiltonian we have removed the terms that induce hopping between different channels, $J_{K} c_{1 \sigma \alpha^{\prime}}^{\dagger} c_{1 \sigma \alpha}$. These and higher order couplings are canceled by two cleverly placed small junctions whose tunneling has been magnetically calibrated to the value $t^{\prime} \simeq-J_{K}$.

\section{G. Kondo lattice}

A slightly less trivial circuit is required to simulate a Kondo lattice that has multiple localized impurities interacting with fermions. There are two different ways of doing it, leading to slightly different physics in each case. A simple concatenation of the circuit in Fig. 2 a will produces a set of impurities coupled by a number of sites with free fermions, equivalent to the model introduced by Paredes ${ }^{27}$ for cold atoms. However, the Kondo lattice as known in the literature ${ }^{22}$ considers multiple impurities coupled to a common conduction band, which can be achieved using the circuit in Fig. 2b. Following Sect. IIC we can write down an effective bosonic Hamiltonian

$$
\begin{aligned}
H & =-J \sum_{\langle i, j\rangle} a_{i \sigma}^{\dagger} a_{j \sigma}-t \sum_{k}\left(b_{k \sigma}^{\dagger} a_{i_{k} \sigma}+\text { H.c }\right) \\
& +\sum_{k} E\left(n_{k \uparrow}^{(b)}, n_{k \downarrow}^{(b)}\right)+\sum_{i \sigma} \mu_{i \sigma} n_{i \sigma}^{(a)}
\end{aligned}
$$

where we have introduced different hard-core bosonic operators for pairs in the conduction band, denoted by $a_{i \sigma}, a_{i \sigma}^{\dagger}$, and $n_{i \sigma}^{(a)}=a_{i \sigma}^{\dagger} a_{i \sigma}$, and for pairs in the impurities, given by $b_{i \sigma}, b_{i \sigma}^{\dagger}$, and $n_{k \sigma}^{(b)}=b_{k \sigma}^{\dagger} b_{k \sigma}$.

This circuit does not have a direct translation into a fermionic Anderson model because for such a quasi-2D structure there is no Jordan-Wigner transformation that preserves the shape of the bosonic Hamiltonian. We will however perform an incomplete Jordan-Wigner transformation which acts only on the conduction arrays, $a_{i \sigma}$, leaving the $b_{k \sigma}$ operators untouched,

$$
\begin{aligned}
& a_{i \uparrow}=c_{i \uparrow}(-1)^{\sum_{j<k} n_{j \uparrow}^{(a)}}, \\
& a_{i \downarrow}=c_{i \downarrow}(-1)^{\sum_{j<k} n_{j \downarrow}^{(a)}+N_{\uparrow}^{(a)}},
\end{aligned}
$$

where $N_{\sigma}^{(a)}=\sum_{i} n_{i \sigma}^{(a)}$. With this unitary map, and working out the second order perturbation theory, one arrives to the standard Kondo lattice model

$$
H_{K L}=-J \sum_{\langle i, j\rangle, \sigma} c_{i \sigma}^{\dagger} c_{j \sigma}+\sum_{k, \sigma, \sigma^{\prime}} \frac{J_{K}}{2} \vec{S}_{k} \cdot \vec{\sigma}_{\alpha \sigma^{\prime}} c_{1 \sigma}^{\dagger} c_{1 \sigma^{\prime}}
$$

Here, $\vec{S}_{k}$ denotes the spin of the $k$-th impurity which is coupled to the site $i_{k}$ and is defined using bosonic operators $\vec{S}_{i_{k}}=\sum_{\sigma, \sigma^{\prime}} \frac{1}{2} \vec{\sigma}_{\alpha \sigma^{\prime}} b_{i_{k} \sigma^{\prime}}^{\dagger} b_{i_{k} \sigma^{\prime}}$. This general notation allows for having less impurities than conduction electron, as well as them being placed on arbitrary positions. The coupling is once more anti-ferromagnetic $J_{K} \sim t^{2} / \epsilon$ and just as tunable as in the case of a single impurity. 


\section{ENERGY SCALES AND DECOHERENCE}

Let us consider what are the experimental challenges for implementing these mappings. We begin with the energy scales of our problem. On the one hand we have free fermions, which hop with a tunneling amplitude $J$ and a Fermi energy $\epsilon_{F} \sim 2 J$. In current experiments with charge qubits $J$ can be roughly of order of a few gigahertz. On the other hand we have the Kondo coupling $J_{K}=$ $t^{2} / \epsilon$, which will be in general smaller. For this coupling to be relevant, the temperature at which the experiment is performed should lay below the Kondo Temperature, $k_{B} T_{K}=\epsilon_{F} \exp \left[1 / J_{K} \rho\left(k_{F}\right)\right]$, where $\rho\left(k_{f}\right)$ is the density of states at the Fermi energy. Since in our case the density of states at half filling $(\mu=0)$ is roughly $\rho\left(k_{f}\right)=\pi / J$, using $t \sim J$, we have $k_{B} T_{K} \sim 2 J \exp (-\epsilon / J \pi)$. This means that, conservatively, for $\epsilon / J=8-10$, the Kondo temperature $T_{K} \sim 40-75 \mathrm{mK}$ would be larger $20 \mathrm{mK}$, the refrigerator temperature of current experiments 14 .

Another important challenge is decoherence, which in our setup arises mainly from charge fluctuations. As is the case of scalable quantum computation, the characterization of decoherence in multi-qubit setups is still an open problem that deserves further investigation. As a guide, we consider the time-scale for the decoherence of a single qubit is $T_{2} \sim 0.5 \mu \mathrm{s} \sqrt{828}$, which is achieved for dispersive readouts. This time-scale gives a frequency of $2 \mathrm{MHz}$ which is well below $J / h \sim 10 \mathrm{GHz}$ and two orders of magnitude away of the lowest frequency $J_{K} / h=t^{2} / \epsilon \sim 100 \mathrm{MHz}$ that we find here. Furthermore, associated to this decoherence rate we can establish an effective temperature of $0.1 \mathrm{mK}$ which is also well below the Kondo temperature.

When compared to other systems, such as cold atoms in optical lattices 4 , the superconducting circuit approach looks as enjoying real advantages. First, the superconducting qubits are not constrained to periodic or quasiperiodic structures. Second and most important, the introduced setup has the potential quality of a good quantum simulator, which is measured by the number of times a particle can hop before the wave function is affected by decoherence. For cold atoms in the lowest band of an optical lattice ${ }^{5}$, coherence times of $500 \mathrm{~ms}$ are to be compared with a hopping rate of $1 \mathrm{kHz}$ between lattice sites, giving a ratio of 500 or better. A similar ratio with superconducting circuits, where the hopping is around 10 $\mathrm{GHz}$, means the decoherence time can be $0.05 \mu \mathrm{s}$, an order of magnitude faster than current experiments 828 . On the other hand, superconducting circuits have the disadvantage of being one-time experiments because the geometry of the couplings cannot be modified in real-time. In addition, it may be complicated to calibrate all islands to reach the sweet spot. However, if we realize that the couplings can be initially switched to zero, this task is as difficult as tuning the individual superconducting qubits in a scalable quantum computer.

\section{MEASUREMENT}

While we have demonstrated that certain quantum circuits can be used to simulate interesting fermionic models, the equivalence between circuit and the Hamiltonian involves the use of Jordan-Wigner transformations (8). Therefore, not all measurements on the quantum circuit will give directly properties of the respective fermionic system. One can envision three kinds of measurements in these systems which do not have this problem. The first one are global transport measurements that mimic past experiments with Josephson junction arrays 17. Since they are based on total particle numbers, they are the same for fermionic and bosonic models.

Next we have spectroscopic measurements and measurement of energy gaps. The coupling between the impurity and the conduction band leads to a binding energy of order $\Delta=k_{B} T_{K}$ given above. This energy can be measured by trying to polarize the impurity with an oscillating voltage applied to the island. We expect this to have an effect either when the potential is of order $\Delta$ or when it oscillates with a frequency of order $\Delta / \hbar$.

The third kind of experiments corresponds to measuring the individual qubits and extracting information about certain correlation functions. In the case of Fig. 11a, the formation of a singlet with the conduction band electrons gives rise to a strong spin correlation in the vicinity of the impurity. The correlation length is of order $\xi=\exp \left[1 / J_{K} \rho\left(k_{F}\right)\right]$ sites and can be seen in the connected correlator as a function of the site $m$

$$
C_{m}=\left\langle S_{0 z} s_{m z}\right\rangle-\left\langle S_{0 z}\right\rangle\left\langle s_{m z}\right\rangle \sim \exp (-m / \xi)
$$

This is known as the Kondo cloud and has not been directly detected by condensed matter setups. In our case, $C_{m}$ can be computed from the statistics of the population difference between islands, $s_{i z}=\left(n_{i \uparrow}-n_{i \uparrow}\right) / 2$, which is best measured using dispersive probes $28 \mid 29$.

\section{CONCLUSIONS}

Summing up, in this paper we have presented a mapping between some superconducting circuits to a family of relevant Hamiltonians describing magnetic impurities in conduction bands. While there are still implementation issues to be solved, they seem less demanding than those of a fully scalable quantum computer implementation with the same technology. We thus expect that our work will motivate further developments, both on the experimental and on the theoretical side, searching better multi-qubit coherence times and other mappings which are based on flux or hybrid qubits.

We thank Vitaly Golovach, Stefan Kehrein, and Jan von Delft for their comments. E.S. acknowledges financial support from DFG SFB 631, EU EuroSQIP projects, and the German Excellence Initiative via the "Nanosystems Initiative Munich (NIM)". 
M.A.M.D. and J.J.G.R. acknowledge financial support from projects FIS2006-04885 (Spanish M.E.C.) and
CAM-UCM/910758. J.J.G.R. acknowledges support from the Ramon y Cajal Program of the M.E.C.
1 R. P. Feynman, Int. J. Theor. Phys. 21, 467 (1982).

2 S. Lloyd, Science 273, 1073 (1996).

3 D. S. Abrams and S. Lloyd, Phys. Rev. Lett. 79, 2586 (1997).

${ }^{4}$ D. Jaksch, C. Bruder, J. I. Cirac, C. W. Gardiner, and P. Zoller, Phys. Rev. Lett. 81, 3108 (1998).

5 M. Greiner, O. Mandel, T. Esslinger, T. W. Hänsch, and I. Bloch, Nature 415, 39 (2002).

${ }^{6}$ V. Bouchiat, D. Vion, P. Joyez, D. Esteve, and M. H. Devoret, Physica Scripta Volume T 76, 165 (1998).

7 Y. Nakamura, Y. A. Pashkin, and J. S. Tsai, Nature 398, 786 (1999).

8 D. Vion, A. Aassime, A. Cottet, P. Joyez, H. Pothier, C. Urbina, D. Esteve, and M. H. Devoret, Science 296, 886 (2002).

9 J. M. Martinis, S. Nam, J. Aumentado, and C. Urbina, Phys. Rev. Lett. 89, 117901 (2002).

10 I. Chiorescu, Y. Nakamura, C. J. P. M. Harmans, and J. E. Mooij, Science 299, 1869 (2003).

11 T. Yamamoto, Y. A. Pashkin, O. Astafiev, Y. Nakamura, and J. S. Tsai, Nature 425, 941 (2003).

12 Y. A. Pashkin, T. Yamamoto, O. Astafiev, Y. Nakamura, D. V. Averin, and J. S. Tsai, Nature 421, 823 (2003).

13 T. Duty, D. Gunnarsson, K. Bladh, and P. Delsing, Phys. Rev. B 69, 140503 (2004).

14 A. Wallraff, D. I. Schuster, A. Blais, L. Frunzio, R.-S. Huang, J. Majer, S. Kumar, S. M. Girvin, and R. J. Schoelkopf, Nature 431, 162 (2004).

15 A. G. Fowler, W. F. Thompson, Z. Yan, A. M. Stephens, B. L. T. Plourde, and F. K. Wilhelm, Physical Review B (Condensed Matter and Materials Physics) 76, 174507 (pages 7) (2007).

16 F. Helmer, M. Mariantoni, A. G. Fowler, J. von Delft,
E. Solano, and F. Marquardt, arXiv:0706.3625v1.

17 A. van Oudenaarden and J. E. Mooij, Phys. Rev. Lett. 76, 4947 (1996).

18 R. Fazio and H. van der Zant, Physics Reports 355, 235 (2001).

19 C. Bruder, R. Fazio, and G. Schön, Annalen der Physik 14, 566 (2005).

20 P. W. Anderson, Phys. Rev. 124, 41 (1961).

21 A. C. Hewson, The Kondo Problem to Heavy Fermions (Cambridge Univ. Press, Cambridge, U.K., 1997).

22 H. Tsunetsugu, M. Sigrist, and K. Ueda, Rev. Mod. Phys. 69, 809 (1997).

23 P. Nozieres and A. Blandin, J. Phys. (Paris) 41, 193 (1980).

24 A. Blais, R.-S. Huang, A. Wallraff, S. M. Girvin, and R. J. Schoelkopf, Phys. Rev. A 69, 062320 (2004).

25 P. Jordan and E. Wigner, Z. Phys. 47, 631 (1928).

26 D. C. Mattis, The Theory of Magnetism I (Springer, Berlin, 1988).

27 B. Paredes, C. Tejedor, and J. I. Cirac, Phys. Rev. A 71, 063608 (2005).

28 A. Wallraff, D. I. Schuster, A. Blais, L. Frunzio, J. Majer, M. H. Devoret, S. M. Girvin, and R. J. Schoelkopf, Phys. Rev. Lett. 95, 060501 (2005).

29 G. Johansson, L. Tornberg, V. S. Shumeiko, and G. Wendin, J. Phys: Condens. Matter 18, S901 (2006).

30 J. R. Schrieffer and P. A. Wolff, Phys. Rev. 149, 491 (1966).

31 That is, belonging to the same junction array.

32 Note that this procedure, though equivalent in the results, differs in the method from 30 , where perturbation theory is carried on in momentum space. 